Article

\title{
On the Generalized Distance Energy of Graphs
}

\author{
Abdollah Alhevaz ${ }^{1, *(\mathbb{D}}$, Maryam Baghipur ${ }^{1}$, Hilal A. Ganie ${ }^{2}$ and Yilun Shang ${ }^{3, *(\mathbb{D}}$ \\ 1 Faculty of Mathematical Sciences, Shahrood University of Technology, Shahrood P.O. Box: 316-3619995161, Iran; \\ maryamb8989@gmail.com \\ 2 Department of Mathematics, University of Kashmir, Srinagar 190006, India; hilahmad1119kt@gmail.com \\ 3 Department of Computer and Information Sciences, Northumbria University, Newcastle NE1 8ST, UK \\ * Correspondence: a.alhevaz@gmail.com (A.A.); yilun.shang@northumbria.ac.uk (Y.S.)
}

Received: 16 November 2019; Accepted: 16 December 2019; Published: 19 December 2019

\begin{abstract}
The generalized distance matrix $D_{\alpha}(G)$ of a connected graph $G$ is defined as $D_{\alpha}(G)=\alpha \operatorname{Tr}(G)+$ $(1-\alpha) D(G)$, where $0 \leq \alpha \leq 1, D(G)$ is the distance matrix and $\operatorname{Tr}(G)$ is the diagonal matrix of the node transmissions. In this paper, we extend the concept of energy to the generalized distance matrix and define the generalized distance energy $E^{D_{\alpha}}(G)$. Some new upper and lower bounds for the generalized distance energy $E^{D_{\alpha}}(G)$ of $G$ are established based on parameters including the Wiener index $W(G)$ and the transmission degrees. Extremal graphs attaining these bounds are identified. It is found that the complete graph has the minimum generalized distance energy among all connected graphs, while the minimum is attained by the star graph among trees of order $n$.
\end{abstract}

Keywords: Generalized distance matrix; distance signless Laplacian matrix; transmission regular graph; energy

MSC: 05C50; 05C12; 15A18

\section{Introduction}

Let $G$ be a connected simple graph. The node (also referred to as vertex) set of graph $G$ is $V(G)=$ $\left\{v_{1}, v_{2}, \ldots, v_{n}\right\}$ and the edge set of $G$ is represented by $E(G) \subseteq V(G) \times V(G)$. Denote by $n=|V(G)|$ the order, i.e., the number of nodes, and $m=|E(G)|$ the size, i.e., the number of edges in $G$. For a node $v \in V(G)$, the neighborhood of $v$, denoted by $N(v)$, consists of all nodes adjacent to it. The number of nodes in $V(G)$ is called the degree of $v$ and is signified by $d_{G}(v)$ or simply $d_{v}$ if the underlying graph $G$ is obvious. A regular graph has all its degrees equal. The complement of the graph $G$, denoted by $\bar{G}$, is the graph with the same vertex set with $G$ but whose edge set consists of the edges not present in $G$. As usual, $K_{n}, K_{s, t}, P_{n}$, and $C_{n}$ denote, respectively, the complete graph on $n$ vertices, the complete bipartite graph on $s+t$ vertices, the path on $n$ vertices, and the cycle on $n$ vertices. The adjacency matrix $A=\left(a_{i j}\right) \in \mathbb{R}^{n \times n}$ is a $(0,1)$-matrix with $(i, j)$-entry being 1 , if $v_{i}$ is adjacent to $v_{j}$ and 0 otherwise. The diagonal matrix containing degrees $d_{i}=d_{G}\left(v_{i}\right)(i=1,2, \ldots, n)$ is denoted by $\operatorname{Deg}(G)=\operatorname{diag}\left(d_{1}, d_{2}, \ldots, d_{n}\right) \in \mathbb{R}^{n \times n}$. The Laplacian matrix and signless Laplacian matrix are defined, respectively, by $L(G)=\operatorname{Deg}(G)-A(G) \in \mathbb{R}^{n \times n}$ and $Q(G)=\operatorname{Deg}(G)+A(G) \in \mathbb{R}^{n \times n}$. Their eigenvalues (also referred to as spectra) are often referred to as Laplacian spectrum and signless Laplacian spectrum, respectively. These two matrices are semi-definite, and the spectra of them are sorted, respectively, as $0=\mu_{n} \leq \mu_{n-1} \leq \cdots \leq \mu_{1}$ and $0 \leq q_{n} \leq q_{n-1} \leq \cdots \leq q_{1}$.

Given two nodes $u, v \in V(G)$, the distance between them is denoted by $d_{u v}$, which is the number of edges along the shortest path between them. The maximum distance between any pair of nodes is 
called the diameter of $G . D(G)=\left(d_{u v}\right)_{u, v \in V(G)} \in \mathbb{R}^{n \times n}$ is the distance matrix. Various spectral properties of $D(G)$ have been reported in, e.g., the survey by Aouchiche and Hansen [1]. Recall that a cut vertex (cut edge) in a connected graph is one whose deletion increases its number of connected components. A vertex-cut set (respectively, edge-cut set) of a connected graph $G$ is a set $S$ of vertices (respectively, a set $L$ of edges) whose removal disconnects $G$ (the number of connected components of $G \backslash S$ (respectively, $G \backslash L$ ) is greater than one). Moreover, a cut ideal of a graph defined in the literature to record the relations between cuts of the graph. There is a relationship between the notions of cut set and cut ideal associated with a graph with the distance matrix of a connected graph. For some literature regarding this relationship, we refer to [2-5].

Given $v \in V(G), \operatorname{Tr}_{G}(v)=\sum_{u \in V(G)} d_{u v}$ is called the transmission of $v$. It is the sum of distances from all nodes to $v$. If, for all $v \in V(G), \operatorname{Tr}_{G}(v)=k$, then the graph $G$ is $k$-transmission regular. The well known Wiener index (also referred to as transmission) of $G$ represents the sum of all individual transmissions, namely $W(G)=\frac{1}{2} \sum_{v \in V(G)} \operatorname{Tr}_{G}(v)$. $\operatorname{Tr}_{G}\left(v_{i}\right)$ is also called the transmission degree and is written as $\operatorname{Tr}_{i}$ for simplicity. Accordingly, the transmission degree sequence is denoted by $\left\{\operatorname{Tr}_{1}, \operatorname{Tr}_{2}, \ldots, \operatorname{Tr}_{n}\right\}$. Define $T_{i}=\sum_{j=1}^{n} d_{i j} T r_{j}$ as the second transmission degree of node $v_{i}$ and $\operatorname{Tr}(G)=\operatorname{diag}\left(\operatorname{Tr}_{1}, \operatorname{Tr}_{2}, \ldots, \operatorname{Tr}_{n}\right) \in \mathbb{R}^{n \times n}$ as the diagonal matrix of node transmissions of $G$. Recent works [6-9] explored the spectral properties of (signless) Laplacian of the distance matrix in connected graphs. Given $G$, its distance Laplacian and distance signless Laplacian are, respectively, defined as $D^{L}(G)=\operatorname{Tr}(G)-D(G) \in \mathbb{R}^{n \times n}$ and $D^{Q}(G)=\operatorname{Tr}(G)+D(G) \in \mathbb{R}^{n \times n}$. Many results have been reported on the spectral properties of $D(G), D^{L}(G)$ and $D^{Q}(G)$ including spectral radius, second largest eigenvalue, and the smallest eigenvalue. For some recent works, we refer to [7-20] (and the references therein).

Recently, in [21], Cui et al. introduced the generalized distance matrix $D_{\alpha}(G)$ as a convex combinations of $\operatorname{Tr}(G)$ and $D(G)$. Namely, $D_{\alpha}(G)=\alpha \operatorname{Tr}(G)+(1-\alpha) D(G)$, where $0 \leq \alpha \leq 1$. Since $D_{0}(G)=D(G), 2 D_{\frac{1}{2}}(G)=D^{Q}(G), D_{1}(G)=\operatorname{Tr}(G)$, and $D_{\alpha}(G)-D_{\beta}(G)=(\alpha-\beta) D^{L}(G)$, any result regarding the spectral properties of generalized distance matrix has its counterpart for each of these particular graph matrices, and these counterparts follow immediately from a single proof. In fact, this matrix reduces to merging the distance spectral and distance (signless) Laplacian spectral theories. Since the eigenvalues of $D_{\alpha}(G)$ are real, we can arrange them as $\partial_{1} \geq \partial_{2} \geq \cdots \geq \partial_{n}$.

The notion of graph energy has its origin in theoretical chemistry and was put forward by Gutman [22]. Let $\lambda_{1}, \lambda_{2}, \ldots, \lambda_{n}$ be the adjacency eigenvalues of $G$. The graph energy of $G$ is defined as $E(G)=\sum_{i=1}^{n}\left|\lambda_{i}\right|$ [23]. Since its inception, graph energy has been extensively studied across chemical and mathematical literature. The upper and lower bounds for $E(G)$ have been reported in some pioneering work (e.g., [24-26]). The work by Gutman [22] signposted a fruitful relationship between graph energy and various eigenvalues of matrices associated to graphs. This leads to a wealth of work on energy-like graph spectral invariant in regards to, e.g., (signless) Laplacian matrix [27,28], Randić matrix [29], and distance matrix [30]. More recent results can be found in [17,18,24,29-33]. In another direction, the graph energy has been extended to digraphs and various energies of digraphs such as energy [34] and skew energy [35] were put forward and extensively studied. This concept was generalized by Nikiforov by defining the energy of any matrix [31]. Many studies, both theory- and application-oriented, have been done along this direction $[24,32,34]$. For example, if $\xi_{1}, \xi_{2}, \ldots, \xi_{n}$ are the eigenvalues of a molecular matrix $M$, the expression $E(M)=\sum_{i=1}^{n}\left|\xi_{i}-\frac{\sum_{j=1}^{n} \tilde{\xi}_{i}}{n}\right|$ was proposed in [36] to facilitate the design of quantitative structure for different types of organic compounds.

Let $\rho_{1}^{D} \geq \rho_{2}^{D} \geq \ldots \geq \rho_{n}^{D}, \rho_{1}^{L} \geq \rho_{2}^{L} \geq \ldots \geq \rho_{n}^{L}$, and $\rho_{1}^{Q} \geq \rho_{2}^{Q} \geq \ldots \geq \rho_{n}^{Q}$ be, respectively, the distance eigenvalues, distance Laplacian eigenvalues, and distance signless Laplacian eigenvalues of graph $G$. 
The distance energy of a graph $G$ was defined as the sum of the absolute values of the distance eigenvalues of $G$ [30]:

$$
E^{D}(G)=\sum_{i=1}^{n}\left|\rho_{i}^{D}\right|
$$

The following fundamental relationships hold

$$
\sum_{i=1}^{n} \rho_{i}^{D}=0 \text { and } \sum_{i=1}^{n}\left(\rho_{i}^{D}\right)^{2}=2 \sum_{1 \leq i<j \leq n}\left(d_{i j}\right)^{2}
$$

For more results on distance energy, we refer the reader to [16]. Analogous to the definition of (signless) Laplacian energy, the concept of distance (signless) Laplacian energy was explored in [12,16,37]. The distance Laplacian energy of a graph $G$ is defined as the mean deviation of the values of the distance Laplacian eigenvalues of $G$ :

$$
E^{L}(G)=\sum_{i=1}^{n}\left|\rho_{i}^{L}-\frac{2 W(G)}{n}\right|
$$

Similarly, the distance signless Laplacian energy can be viewed as the mean deviation of the values of the distance signless Laplacian eigenvalues of $G$ :

$$
E^{Q}(G)=\sum_{i=1}^{n}\left|\rho_{i}^{Q}-\frac{2 W(G)}{n}\right|
$$

Recent results on $E^{L}(G)$ and $E^{Q}(G)$ can be found (see, e.g., [12,16,38]).

Motivated by the above works, we here introduce an energy-like quantity based on the generalized distance matrix $D_{\alpha}(G)$. We define the auxiliary eigenvalues $\Theta_{i}$, corresponding to the generalized distance eigenvalues, as $\Theta_{i}=\partial_{i}-\frac{2 \alpha W(G)}{n}$. Analogous to the definitions of distance Laplacian energy $E^{L}(G)$ and distance signless Laplacian energy $E^{Q}(G)$, the generalized distance energy of $G$ can be thought of as the mean deviation of the values of the generalized distance eigenvalues of $G$, namely,

$$
E^{D_{\alpha}}(G)=\sum_{i=1}^{n}\left|\partial_{i}-\frac{2 \alpha W(G)}{n}\right|=\sum_{i=1}^{n}\left|\Theta_{i}\right|
$$

Therefore, we have $\sum_{i=1}^{n} \Theta_{i}=0$. In addition, since $\sum_{i=1}^{n} \partial_{i}=2 \alpha W(G)$ and $\sum_{i=1}^{n} \partial_{i}^{2}=\operatorname{trace}\left[D_{\alpha}(G)\right]^{2}=$ $2(1-\alpha)^{2} \sum_{1 \leq i<j \leq n}^{n}\left(d_{i j}\right)^{2}+\alpha^{2} \sum_{i=1}^{n} \operatorname{Tr}_{i}^{2}$, thanks to the definition of $\Theta_{i}$, we see that $\sum_{i=1}^{n} \Theta_{i}^{2}=2 \zeta$, where

$$
\zeta=(1-\alpha)^{2} \sum_{1 \leq i<j \leq n}^{n}\left(d_{i j}\right)^{2}+\frac{\alpha^{2}}{2} \sum_{i=1}^{n} \operatorname{Tr}_{i}^{2}-\frac{2 \alpha^{2} W^{2}(G)}{n}
$$

Moreover, $E^{D_{0}}(G)=E^{D}(G)$ and $2 E^{D^{\frac{1}{2}}}(G)=E^{Q}(G)$. This implies that the concept of generalized distance energy of a graph $G$ merges the theories of distance energy and distance signless Laplacian energy. Hence, it is desirable to tackle the quantity $E^{D_{\alpha}}(G)$ and its related structural and algebraic properties including upper and lower bounds, and the parameter (i.e., $\alpha$ ) dependency on the graph topology. 
The remainder of the paper is organized as follows. Some preliminary results and useful lemmas are presented in Section 2. The main results, namely the upper bounds and lower bounds for the generalized distance energy $E^{D_{\alpha}}(G)$ are presented, respectively, in Sections 3 and 4 . The bounds link important graph parameters such as the Wiener index $W(G)$ and the transmission degrees. The extremal graph attaining these bounds are also characterized. We show that the generalized distance energy $E^{D_{\alpha}}(G)$ can be obtained from the corresponding distance energy $E^{D}(G)$ for the transmission regular graphs. Among other results, we prove that, if $G$ is a connected graph of order $n \geq 2$ and $\alpha \in[0,1)$, then

$$
E^{D_{\alpha}}(G) \geq 2(1-\alpha)(n-1),
$$

where the equality holds if and only if $G \cong K_{n}$. It is revealed from this result that the complete graphs attain the minimum generalized distance energy over all connected graphs. We also prove that, if $T$ is a tree of order $n \geq 4$ and $\alpha \in[0,1)$, then

$$
E^{D_{\alpha}}(T) \geq(2-3 \alpha) n+8 \alpha-4+\sqrt{(\alpha-2)^{2} n^{2}+4(n-1)(2 \alpha-3)}-\frac{4 \alpha}{n},
$$

where the equality holds if and only if $T \cong K_{1, n-1}$. It is revealed from this result that the minimum generalized distance energy within trees are attained by the star graphs. We conclude in Section 5 .

\section{Preliminary Results}

In this section, we summarize some useful results, which become building blocks of the main results. The following lemma can be found in [39].

Lemma 1. ([39]). Let $G$ be a tree of order $n \geq 4$. If $0 \leq \alpha<1$, then,

$$
\partial(G) \geq \frac{1}{2}\left((\alpha+2) n-4+\sqrt{(2-\alpha)^{2} n^{2}+4(n-1)(2 \alpha-3)}\right),
$$

where the equality holds if and only if $G \cong K_{1, n-1}$.

Lemma 2. ([21]). Let the transmission degree sequence be $\left\{\operatorname{Tr}_{1}, \operatorname{Tr}_{2}, \ldots, \operatorname{Tr}_{n}\right\}$. Let the second transmission degree sequence be $\left\{T_{1}, T_{2}, \ldots, T_{n}\right\}$. We have

$$
\partial(G) \geq \sqrt{\frac{\sum_{i=1}^{n}\left(\alpha \operatorname{Tr}_{i}^{2}+(1-\alpha) T_{i}\right)^{2}}{\sum_{i=1}^{n} \operatorname{Tr}_{i}^{2}}} .
$$

Moreover, if $\frac{1}{2} \leq \alpha \leq 1$, the equality holds if and only if $G$ is transmission regular.

Lemma 3. ([21]). If $G$ is a connected graph of order $n$, then

$$
\partial(G) \geq \frac{2 W(G)}{n}
$$

where the equality holds if and only if $G$ is transmission regular.

Lemma 4. ([21]). Let $G$ be a connected graph of order $n$ and $\frac{1}{2} \leq \alpha \leq 1$. If $G^{\prime}$ is a connected graph obtained from $G$ by removing one edge, then $\partial_{i}\left(G^{\prime}\right) \geq \partial_{i}(G)$, for all $1 \leq i \leq n$.

For a matrix $M$, the definition of $E(M)$ is given in [40] (see also the Introduction). 
Lemma 5. ([40]). Let $A, B \in \mathbb{R}^{n \times n}$ and let $C=A+B$. Then

$$
E(C) \leq E(A)+E(B),
$$

where the equality holds if and only if there exists an orthogonal matrix $P$ such that $P A$ and $P B$ are both positive semi-definite matrices.

The following fundamental lemma regarding positive semidefinite matrix can be found in [41].

Lemma 6. ([41]). If $M=\left(m_{i j}\right)$ is a positive semidefinite matrix of order $n$ with $m_{i i}=0$ for some $i$, then $m_{i j}=0=m_{j i}$, for $j=1,2, \ldots, n$.

The polar decomposition theorem is given in [42].

Lemma 7. Let $M \in \mathbb{R}^{n \times n}$. There exist positive semidefinite matrices $X, Y \in \mathbb{R}^{n \times n}$ and orthogonal matrices $P, F \in \mathbb{R}^{n \times n}$ such that $M=P X=Y F$. Moreover, the matrices $X=\left(M^{T} M\right)^{\frac{1}{2}}$ and $Y=\left(M M^{T}\right)^{\frac{1}{2}}$ are unique. The matrices $P$ and $F$ are uniquely determined if and only if $M$ is non-singular.

The following lemma characterizes the graphs with a pair of distinct generalized distance eigenvalues. It can be proved similarly as ([43] Lemma 2.10).

Lemma 8. Take a connected graph $G$. It contains a pair of distinct $D_{\alpha}(G)$ eigenvalues if and only if $G$ is a complete graph.

For any non-negative integers $i, j, k$, a connected graph $G$ is said to be distance regular if for any two nodes $u, v \in V(G)$ with $d(u, v)=k$, the number of nodes $w \in V(G)$ satisfying $d(u, w)=i$ and $d(v, w)=j$ is independent of $u$ and $v$. A distance regular graph is always transmission regular [15]. The following characterization for the distance regular graphs with a single positive distance eigenvalue can be found in [44].

Lemma 9. A distance regular graph $G$ has a single positive distance eigenvalue if and only if $G$ is isomorphic to one of the following graphs: a Doob graph, a double odd graph, a Johnson graph, a cocktail party graph, a polygon, a halved cube, a Hamming graph, the Petersen graph, the Schläfli graph, the Gosset graph, the dodecahedron, the icosahedron, and one of the three Chang graphs.

\section{Upper Bounds for the Generalized Distance Energy}

In this section, we present some upper bounds for the generalized distance energy $E^{D_{\alpha}}(G)$ for $\alpha \in[0,1]$ of a connected graph $G$. These bounds links some important graph parameters such as the Wiener index $W(G)$ and the transmission degrees. The extremal graph attaining these bounds are delineated.

The following result gives an upper bound for the generalized distance energy $E^{D_{\alpha}}(G)$ connecting the distance energy $E^{D}(G)$, the transmission degrees, and the Wiener index $W(G)$.

Theorem 1. Let $G$ be a connected graph with transmission degree sequence $\left\{\operatorname{Tr}_{1}, \operatorname{Tr}_{2}, \ldots, \operatorname{Tr}_{n}\right\}$. We have

$$
E^{D_{\alpha}}(G) \leq(1-\alpha) E^{D}(G)+\sum_{i=1}^{n}\left|\alpha \operatorname{Tr}_{i}-\frac{2 \alpha W(G)}{n}\right|
$$


In the case of $\alpha=0,1$, the equality in Equation (2) invariably occurs. For $0<\alpha<1$, equality in Equation (2) occurs if and only if $G$ is a transmission regular graph.

Proof. Let $D_{\alpha}(G)$ be the generalized distance matrix of the connected graph $G$ of order $n$. We have

$$
\left(D_{\alpha}(G)-\frac{2 \alpha W(G)}{n} I_{n}\right)=(1-\alpha) D(G)+\left(\alpha \operatorname{Tr}(G)-\frac{2 \alpha W(G)}{n} I_{n}\right) .
$$

Apply Lemma 5, and using the fact that $\alpha \operatorname{Tr}(G)-\frac{2 \alpha W(G)}{n} I_{n}$ is a diagonal matrix whose eigenvalues are $\alpha \operatorname{Tr}_{i}-\frac{2 \alpha W(G)}{n}, i=1,2, \ldots, n$, the inequality in Equation (2) follows.

If $\alpha=0$, then it is clear that $D_{\alpha}(G)=D(G)$ and thus the equality in Equation (2) always hold. If $\alpha=1$, then $D_{\alpha}(G)=\operatorname{Tr}(G)$ and thus equality always occurs. Now, suppose that $0<\alpha<1$. If $G$ is a transmission regular graph, then $2 W(G)=n T r_{1}$ and thus the equality in Equation (2) holds. Suppose that equality holds in Equation (2). We show that $G$ is a transmission regular graph. Assume on the contrary that $G$ is not a transmission regular graph. Then, $\operatorname{Tr}_{\max }=\operatorname{Tr}_{1}>\frac{2 W(G)}{n}$. For $i=1,2, \ldots, n$, let $a_{i}=\alpha \operatorname{Tr}_{i}-\frac{2 \alpha W(G)}{n}$. Then, $a_{1}=\alpha\left(\operatorname{Tr}_{1}-\frac{2 W(G)}{n}\right)>0$ as $\alpha>0$. Since equality occurs in Equation (2), it follows from Lemma 5 that there exists an orthogonal matrix $P$ satisfying $X=P\left(\alpha \operatorname{Tr}(G)-\frac{2 \alpha W(G)}{n} I_{n}\right)$ and $Y=P((1-\alpha) D(G))$ are both positive semi-definite matrices. This gives that the matrices $P^{T} X$ and $P^{T} Y$ (as $P$ is orthogonal, hence $P^{T}=P^{-1}$ ) are the polar decompositions of the matrices $\alpha \operatorname{Tr}(G)-\frac{2 \alpha W(G)}{n} I_{n}$ and $(1-\alpha) D(G)$, respectively. Using, Lemma 7 and the fact that the matrices $\alpha \operatorname{Tr}(G)-\frac{2 \alpha W(G)}{n} I_{n}$ and $(1-\alpha) D(G)$ are symmetric matrices, we get $X=\left|\alpha \operatorname{Tr}(G)-\frac{2 \alpha W(G)}{n} I_{n}\right|=\operatorname{diag}\left(\left|a_{1}\right|,\left|a_{2}\right|, \ldots,\left|a_{n}\right|\right)$ and $Y=|(1-\alpha) D(G)|$. Let $P^{T}=\left(\begin{array}{cccc}p_{11} & p_{12} & \cdots & p_{1 n} \\ p_{21} & p_{22} & \cdots & p_{2 n} \\ \vdots & \vdots & \cdots & \vdots \\ p_{n 1} & p_{n 2} & \cdots & p_{n n}\end{array}\right)$ and $D(G)=\left(\begin{array}{cccc}0 & d_{12} & \cdots & d_{1 n} \\ d_{12} & 0 & \cdots & d_{2 n} \\ \vdots & \vdots & \cdots & \vdots \\ d_{1 n} & d_{2 n} & \cdots & 0\end{array}\right)$. We have $P^{T} X=\alpha \operatorname{Tr}(G)-\frac{2 \alpha W(G)}{n} I_{n}$, giving that $\left(\begin{array}{cccc}\left|a_{1}\right| p_{11} & \left|a_{2}\right| p_{12} & \cdots & \left|a_{n}\right| p_{1 n} \\ \left|a_{1}\right| p_{21} & \left|a_{2}\right| p_{22} & \cdots & \left|a_{n}\right| p_{2 n} \\ \vdots & \vdots & \cdots & \vdots \\ \left|a_{1}\right| p_{n 1} & \left|a_{2}\right| p_{n 2} & \cdots & \left|a_{n}\right| p_{n n}\end{array}\right)=\left(\begin{array}{cccc}a_{1} & 0 & \cdots & 0 \\ 0 & a_{2} & \cdots & 0 \\ \vdots & \vdots & \cdots & \vdots \\ 0 & 0 & \cdots & a_{n}\end{array}\right)$. Using equality of matrices, the equality at first column gives $p_{11}=1$ and $p_{j 1}=0$, for all $j=2,3, \ldots, n$. From this, it follows that $P=\left(\begin{array}{cccc}1 & 0 & \cdots & 0 \\ p_{12} & p_{22} & \cdots & p_{n 2} \\ \vdots & \vdots & \cdots & \vdots \\ p_{1 n} & p_{2 n} & \cdots & p_{n n}\end{array}\right)$. Now, $Y=(1-\alpha) P D(G)$ gives that $Y=(1-\alpha)\left(\begin{array}{cccc}1 & 0 & \cdots & 0 \\ p_{12} & p_{22} & \cdots & p_{n 2} \\ \vdots & \vdots & \cdots & \vdots \\ p_{1 n} & p_{2 n} & \cdots & p_{n n}\end{array}\right)\left(\begin{array}{cccc}0 & d_{12} & \cdots & d_{1 n} \\ d_{12} & 0 & \cdots & d_{2 n} \\ \vdots & \vdots & \cdots & \vdots \\ d_{1 n} & d_{2 n} & \cdots & 0\end{array}\right)=(1-\alpha)\left(\begin{array}{cccc}0 & d_{12} & \cdots & d_{1 n} \\ c_{21} & c_{22} & \cdots & c_{2 n} \\ \vdots & \vdots & \cdots & \vdots \\ c_{n 1} & c_{n 2} & \cdots & c_{n n}\end{array}\right)$, where $c_{i j}$ is the product of $i$ th row of $P$ with the $j$ th column of $D(G)$, for $i=2,3, \ldots, n$ and $j=1,2, \ldots, n$. Since the matrix $Y$ is a positive semidefinite matrix, it follows from Lemma 6 that $d_{1 j}=0$, for all $j=2,3, \ldots, n$. This contracts the fact that $G$ is a connected graph. That completes the proof. 
It is clear from Theorem 1 that any upper bound for $E^{D}(G)$ is also an upper bound for $E^{D_{\alpha}}(G)$. The following result gives that the generalized distance energy $E^{D_{\alpha}}(G)$ can be obtained from the corresponding distance energy $E^{D}(G)$ for the transmission regular graphs.

Theorem 2. Let $G$ be a transmission regular graph of order $n$ and $\alpha \in[0,1]$. Then, $E^{D_{\alpha}}(G)=(1-\alpha) E^{D}(G)$.

Proof. Let $\delta_{1}, \ldots, \delta_{n}$ be the distance eigenvalues of the graph $G$. Suppose that $G$ is a $k$-transmission regular graph, then $\operatorname{Tr}(G)=k I_{n}$ and so

$$
D_{\alpha}=\alpha \operatorname{Tr}(G)+(1-\alpha) D(G)=\alpha k I_{n}+(1-\alpha) D(G) .
$$

From this, it follows that the generalized distance spectrum of $G$ turns out to be $\{\alpha k+(1-$ $\left.\alpha) \delta_{1}, \ldots, \alpha k+(1-\alpha) \delta_{n}\right\}$. Using this, we have $E^{D_{\alpha}}(G)=(1-\alpha) E^{D}(G)$.

The following result presents an upper bound for the generalized distance energy $E^{D_{\alpha}}(G)$, connecting the Wiener index $W(G)$ as well as the transmission degrees.

Theorem 3. Let $G$ be a connected graph of order $n \geq 3$ and $\alpha \leq 1-\frac{n}{2 W(G)}$. Then,

$$
\begin{aligned}
E^{D_{\alpha}}(G) & \leq 2(1-\alpha)^{2} \sum_{1 \leq i<j \leq n}\left(d_{i j}\right)^{2}+\alpha^{2} \sum_{i=1}^{n} \operatorname{Tr}_{i}^{2}+\ln \left(\frac{2 \alpha(1-\alpha) W(G)}{n \Gamma}\right) \\
& -\frac{2 \alpha W(G)}{n^{2}}(2 \alpha n W(G)+2 \alpha W(G)-4 W(G)+n)+\frac{2 W(G)}{n}-\frac{4 W^{2}(G)}{n^{2}},
\end{aligned}
$$

where $\Gamma=\left|\operatorname{det}\left(D_{\alpha}(G)-\frac{2 \alpha W(G)}{n} I\right)\right|$. The equality in Equation (3) holds if and only if $\alpha=0$ and $G \cong K_{n}$ or $G$ is a $k$-transmission regular graph with three distinct generalized distance eigenvalues, namely $k, \alpha k+1$ and $\alpha k-1$.

Proof. Consider a connected graph $G$ of order $n$ and size $m$. Let $\partial_{1} \geq \partial_{2} \geq \cdots \geq \partial_{n}$ be the generalized distance eigenvalues of $G$. Consider the function

$$
f(x)=\left(x-\frac{2 \alpha W(G)}{n}\right)^{2}-\left(x-\frac{2 \alpha W(G)}{n}\right)-\ln \left(x-\frac{2 \alpha W(G)}{n}\right), \quad x-\frac{2 \alpha W(G)}{n}>0
$$

for which

$$
f^{\prime}(x)=2\left(x-\frac{2 \alpha W(G)}{n}\right)-1-\frac{1}{x-\frac{2 \alpha W(G)}{n}} .
$$


Thus, $f(x)$ is increasing over $x-\frac{2 \alpha W(G)}{n} \geq 1$ and decreasing over $0<x-\frac{2 \alpha W(G)}{n} \leq 1$. Thus, $f(x) \geq f\left(\frac{2 \alpha W(G)}{n}+1\right)=0$ and $x-\frac{2 \alpha W(G)}{n} \leq\left(x-\frac{2 \alpha W(G)}{n}\right)^{2}-\ln \left(x-\frac{2 \alpha W(G)}{n}\right)$ for $x-\frac{2 \alpha W(G)}{n}>0$, where the equality holds if and only if $x-\frac{2 \alpha W(G)}{n}=1$. Using this result, we arrive at

$$
\begin{aligned}
E^{D_{\alpha}}(G) & =\partial_{1}-\frac{2 \alpha W(G)}{n}+\sum_{i=2}^{n}\left|\partial_{i}-\frac{2 \alpha W(G)}{n}\right| \\
& \leq \partial_{1}-\frac{2 \alpha W(G)}{n}+\sum_{i=2}^{n}\left(\left(\partial_{i}-\frac{2 \alpha W(G)}{n}\right)^{2}-\ln \left|\partial_{i}-\frac{2 \alpha W(G)}{n}\right|\right) \\
& =\partial_{1}-\frac{2 \alpha W(G)}{n}+2(1-\alpha)^{2} \sum_{1 \leq i<j \leq n}\left(d_{i j}\right)^{2}+\alpha^{2} \sum_{i=1}^{n} \operatorname{Tr}_{i}^{2}-\partial_{1}^{2}+\frac{4 \alpha^{2} W^{2}(G)}{n^{2}}(n-1) \\
& -\frac{4 \alpha W(G)}{n}\left(2 \alpha W(G)-\partial_{1}\right)-\ln \prod_{i=1}^{n}\left|\partial_{i}-\frac{2 \alpha W(G)}{n}\right|+\ln \left(\partial_{1}-\frac{2 \alpha W(G)}{n}\right) \\
& =2(1-\alpha)^{2} \sum_{1 \leq i<j \leq n}\left(d_{i j}\right)^{2}+\alpha^{2} \sum_{i=1}^{n} \operatorname{Tr}_{i}^{2}-\frac{2 \alpha W(G)}{n^{2}}(2 n \alpha W(G)+2 \alpha W(G)+n) \\
& +\partial_{1}\left(\frac{4 \alpha W(G)}{n}+1\right)-\partial_{1}^{2}-\ln \Gamma+\ln \left(\partial_{1}-\frac{2 \alpha W(G)}{n}\right) .
\end{aligned}
$$

From Lemma 3, it can be seen that $\partial_{1} \geq \frac{2 W(G)}{n}$. Consider the function

$$
\begin{aligned}
g(x) & =2(1-\alpha)^{2} \sum_{1 \leq i<j \leq n}\left(d_{i j}\right)^{2}+\alpha^{2} \sum_{i=1}^{n} \operatorname{Tr}_{i}^{2}-\frac{2 \alpha W(G)}{n^{2}}(2 n \alpha W(G)+2 \alpha W(G)+n) \\
& +x\left(\frac{4 \alpha W(G)}{n}+1\right)-x^{2}-\ln \Gamma+\ln \left(x-\frac{2 \alpha W(G)}{n}\right) .
\end{aligned}
$$

It is straightforward to see that $g(x)$ is increasing when $0<x-\frac{2 \alpha W(G)}{n} \leq 1$ and decreasing when $x-\frac{2 \alpha W(G)}{n} \geq 1$. Since for $\alpha \leq 1-\frac{n}{2 W(G)}$, we have $x-\frac{2 \alpha W(G)}{n} \geq(1-\alpha) \frac{2 W(G)}{n} \geq 1$, it follows that

$$
\begin{aligned}
g(x) \leq g\left(\frac{2 W(G)}{n}\right) & =2(1-\alpha)^{2} \sum_{1 \leq i<j \leq n}\left(d_{i j}\right)^{2}+\alpha^{2} \sum_{i=1}^{n} \operatorname{Tr}_{i}^{2}-\ln \Gamma+\ln \left(\frac{2 \alpha W(G)}{n}(1-\alpha)\right) \\
& -\frac{2 \alpha W(G)}{n^{2}}(2 \alpha n W(G)+2 \alpha W(G)-4 W(G)+n)+\frac{2 W(G)}{n}-\frac{4 W^{2}(G)}{n^{2}} .
\end{aligned}
$$

Combining this with Equation (5), we arrive at Equation (3). This completes the first part. Next, suppose equality holds in Equation (3). Then, $\partial_{1}=\frac{2 W(G)}{n}$ and thus, by Lemma 3, G is a transmission regular graph. From the equality in Equation (4), we get $\left|\partial_{i}-\frac{2 \alpha W(G)}{n}\right|=1$, for $i=2,3, \ldots, n$. This gives that $\left|\partial_{i}-\frac{2 \alpha W(G)}{n}\right|$ can have at most two distinct values and we arrive at the following. If $\partial_{i}-\frac{2 \alpha W(G)}{n}=1$, for all $i=2,3, \ldots, n$, then $\partial_{i}=1+\frac{2 \alpha W(G)}{n}$, for all $i=2,3, \ldots, n$, giving that $G$ has two distinct generalized distance eigenvalues, namely $\partial_{1}=\frac{2 W(G)}{n}$ and $\partial_{i}=1+\frac{2 \alpha W(G)}{n}$. Thus, by Lemma 8, equality occurs for complete graph $K_{n}$, provided that the generalized distance eigenvalues of $K_{n}$ are $n-1$ with multiplicity 1 and $\alpha(n-1)+1$ with multiplicity $n-1$. Since the generalized distance eigenvalues of $K_{n}$ are $n-1$ with multiplicity 1 and $\alpha n-1$ with multiplicity $n-1$, it follows that equality does not hold in this case. If $\partial_{i}-\frac{2 \alpha W(G)}{n}=-1$, for all $i=2,3, \ldots, n$. Then, $\partial_{i}=\frac{2 \alpha W(G)}{n}-1$, for all $i=2,3, \ldots, n$, giving that $G$ has 
two distinct generalized distance eigenvalues, namely $\partial_{1}=\frac{2 W(G)}{n}$ and $\partial_{i}=\frac{2 \alpha W(G)}{n}-1$. Thus, by Lemma 8 , equality occurs for complete graph $K_{n}$, provided that the generalized distance eigenvalues of $K_{n}$ are $n-1$ with multiplicity 1 and $\alpha(n-1)-1$ with multiplicity $n-1$. Which is true for $\alpha=0$, giving that equality occurs in this case for $\alpha=0$ and if and only if $G \cong K_{n}$. For the remaining case, let for some $t$, we have $\partial_{i}-\frac{2 \alpha W(G)}{n}=1$, for $i=2,3, \ldots, t$ and $\partial_{i}-\frac{2 \alpha W(G)}{n}=-1$, for $i=t+1, \ldots, n$. This gives that $G$ is transmission regular graph with three distinct generalized eigenvalues, namely $\partial_{1}=\frac{2 W(G)}{n}$, with multiplicity $1, \partial_{i}=1+\alpha \partial_{1}$, with multiplicity $t-1$ and $\partial_{i}=\alpha \partial_{1}-1$, with multiplicity $n-t$. Conversely, if $G \cong K_{n}$, then $\partial_{1}=n-1, \partial_{i}=\alpha n-1$, for $i=2,3, \ldots, n$ and $\frac{2 \alpha W\left(K_{n}\right)}{n}=\alpha(n-1)$. It can be seen that equality occurs in (3). On the other hand, if $G$ is a transmission regular graph with three distinct generalized distance eigenvalues, namely $\partial_{1}, \alpha \partial_{1}+1$ and $\alpha \partial_{1}-1$, then equality holds.

An alternative upper bound for the generalized distance energy $E^{D_{\alpha}}(G)$ is given as follows.

Theorem 4. Let $G$ be a connected graph of order $n \geq 3$ and $\alpha \leq \frac{n-2}{n-1}$. Then,

$$
\begin{aligned}
& E^{D_{\alpha}}(G) \leq\left(\frac{4 \alpha W(G)}{n}+1\right)\left(\sqrt{\frac{\sum_{i=1}^{n}\left(\alpha \operatorname{Tr}_{i}^{2}+(1-\alpha) T_{i}\right)^{2}}{\sum_{i=1}^{n} \operatorname{Tr}_{i}^{2}}}\right)-\left(\frac{\sum_{i=1}^{n}\left(\alpha \operatorname{Tr}_{i}^{2}+(1-\alpha) T_{i}\right)^{2}}{\sum_{i=1}^{n} \operatorname{Tr}_{i}^{2}}\right) \\
+ & \ln \left(\frac{\xi}{\Gamma}\right)+2(1-\alpha)^{2} \sum_{1 \leq i<j \leq n}\left(d_{i j}\right)^{2}+\alpha^{2} \sum_{i=1}^{n} \operatorname{Tr}_{i}^{2}-\frac{2 \alpha W(G)}{n^{2}}(2 n \alpha W(G)+2 \alpha W(G)+n),
\end{aligned}
$$

where $\Gamma=\left|\operatorname{det}\left(D_{\alpha}(G)-\frac{2 \alpha W(G)}{n} I\right)\right|$ and $\xi=\sqrt{\frac{\sum_{i=1}^{n}\left(\alpha T r_{i}^{2}+(1-\alpha) T_{i}\right)^{2}}{\sum_{i=1}^{n} T r_{i}^{2}}}-\frac{2 \alpha W(G)}{n}$. The equality in Equation (6) holds if and only if $\alpha=0$ and $G \cong K_{n}$ or $G$ is a $k$-transmission regular graph with three distinct generalized distance eigenvalues, namely $k, \alpha k+1$ and $\alpha k-1$.

Proof. Arguing similarly as in the proof of Theorem 3 yields

$$
\begin{aligned}
E^{D_{\alpha}}(G) & \leq 2(1-\alpha)^{2} \sum_{1 \leq i<j \leq n}\left(d_{i j}\right)^{2}+\alpha^{2} \sum_{i=1}^{n} \operatorname{Tr}_{i}^{2}-\frac{2 \alpha W(G)}{n^{2}}(2 n \alpha W(G)+2 \alpha W(G)+n) \\
& +\partial_{1}\left(\frac{4 \alpha W(G)}{n}+1\right)-\partial_{1}^{2}-\ln \Gamma+\ln \left(\partial_{1}-\frac{2 \alpha W(G)}{n}\right) .
\end{aligned}
$$

In view of Lemma $2, \partial_{1} \geq \sqrt{\frac{\sum_{i=1}^{n}\left(\alpha T r_{i}^{2}+(1-\alpha) T_{i}\right)^{2}}{\sum_{i=1}^{n} T r_{i}^{2}}}$. In addition,

$$
\begin{aligned}
g(x) & =2(1-\alpha)^{2} \sum_{1 \leq i<j \leq n}\left(d_{i j}\right)^{2}+\alpha^{2} \sum_{i=1}^{n} \operatorname{Tr}_{i}^{2}-\frac{2 \alpha W(G)}{n^{2}}(2 n \alpha W(G)+2 \alpha W(G)+n) \\
& +x\left(\frac{4 \alpha W(G)}{n}+1\right)-x^{2}-\ln \Gamma+\ln \left(x-\frac{2 \alpha W(G)}{n}\right),
\end{aligned}
$$


is increasing when $0<x-\frac{2 \alpha W(G)}{n} \leq 1$ and decreasing when $x-\frac{2 \alpha W(G)}{n} \geq 1$. We have $\partial_{1} \geq$ $\sqrt{\frac{\sum_{i=1}^{n}\left(\alpha T_{i}^{2}+(1-\alpha) T_{i}\right)^{2}}{\sum_{i=1}^{n} \operatorname{Tr}_{i}^{2}}} \geq \frac{2 W(G)}{n}$, since, we know $\sum_{i=1}^{n} T_{i}=\sum_{i=1}^{n} \operatorname{Tr}_{i}^{2}$, hence, by Cauchy-Schwartz inequality, $\left(\sum_{i=1}^{n} T_{i}\right)^{2} \leq n \sum_{i=1}^{n} T_{i}^{2}$ and $\left(\sum_{i=1}^{n} T r_{i}\right)^{2} \leq n \sum_{i=1}^{n} \operatorname{Tr}_{i}^{2}$. Then, we get,

$$
\begin{aligned}
\partial_{1} & \geq \sqrt{\frac{\sum_{i=1}^{n}\left(\alpha \operatorname{Tr}_{i}^{2}+(1-\alpha) T_{i}\right)^{2}}{\sum_{i=1}^{n} \operatorname{Tr}_{i}^{2}}} \geq \sqrt{\frac{\left(\sum_{i=1}^{n}\left(\alpha \operatorname{Tr}_{i}^{2}+(1-\alpha) T_{i}\right)\right)^{2}}{n \sum_{i=1}^{n} T r_{i}^{2}}} \\
& =\sqrt{\frac{\left(\sum_{i=1}^{n} \operatorname{Tr}_{i}^{2}\right)^{2}}{n \sum_{i=1}^{n} \operatorname{Tr}_{i}^{2}}}=\sqrt{\frac{\sum_{i=1}^{n} T_{i}^{2}}{n}} \geq \sqrt{\frac{\left(\sum_{i=1}^{n} T r_{i}\right)^{2}}{n^{2}}}=\frac{2 W(G)}{n} .
\end{aligned}
$$

Therefore, for $\alpha \leq \frac{n-2}{n-1}$, we have $x-\frac{2 \alpha W(G)}{n} \geq(1-\alpha) \frac{2 W(G)}{n} \geq 1$. Then,

$$
\begin{aligned}
& g\left(\sqrt{\frac{\sum_{i=1}^{n}\left(\alpha \operatorname{Tr}_{i}^{2}+(1-\alpha) T_{i}\right)^{2}}{\sum_{i=1}^{n} \operatorname{Tr}_{i}^{2}}}\right) \leq\left(\frac{4 \alpha W(G)}{n}+1\right)\left(\sqrt{\frac{\sum_{i=1}^{n}\left(\alpha \operatorname{Tr}_{i}^{2}+(1-\alpha) T_{i}\right)^{2}}{\sum_{i=1}^{n} \operatorname{Tr}_{i}^{2}}}\right)-\ln \Gamma \\
- & \left(\frac{\sum_{i=1}^{n}\left(\alpha \operatorname{Tr}_{i}^{2}+(1-\alpha) T_{i}\right)^{2}}{\sum_{i=1}^{n} \operatorname{Tr}_{i}^{2}}\right)+\ln \left(\sqrt{\frac{\sum_{i=1}^{n}\left(\alpha \operatorname{Tr}_{i}^{2}+(1-\alpha) T_{i}\right)^{2}}{\sum_{i=1}^{n} \operatorname{Tr}_{i}^{2}}}-\frac{2 \alpha W(G)}{n}\right) \\
+ & 2(1-\alpha)^{2} \sum_{1 \leq i<j \leq n}\left(d_{i j}\right)^{2}+\alpha^{2} \sum_{i=1}^{n} \operatorname{Tr}_{i}^{2}-\frac{2 \alpha W(G)}{n^{2}}(2 n \alpha W(G)+2 \alpha W(G)+n) .
\end{aligned}
$$

Combining this with Equation (7), we arrive at Equation (6). The rest of the proof can be done similarly as in Theorem 3, and is omitted here.

\section{Lower Bounds for the Generalized Distance Energy}

In this section, some new lower bounds for the generalized distance energy $E^{D_{\alpha}}(G)$ of a connected graph $G$ are presented. These bounds connects some interesting graph parameters such as the Wiener index $W(G)$ and the transmission degrees. As in the case of upper bounds, the extremal graphs are derived. The complete graphs are shown to have the minimal generalized distance energy among all connected graphs, while the star graphs have the minimal generalized distance energy among all trees.

The following result gives a lower bound for generalized distance energy $E^{D_{\alpha}}(G)$ through the Wiener index $W(G)$.

Theorem 5. Let $G$ be a connected graph of order $n \geq 2$ and let $\alpha \in[0,1)$. Then,

$$
E^{D_{\alpha}}(G) \geq 4(1-\alpha) \frac{W(G)}{n},
$$

where the equality holds if and only if $G$ is a transmission regular graph with one positive distance eigenvalue. 
Proof. Let $G$ be a connected graph of order $n$. Suppose that $t$ is the integer such that $\partial_{t} \geq \frac{2 \alpha W(G)}{n}$ and $\partial_{t+1}<\frac{2 \alpha W(G)}{n}$. Then, by the definition of generalized distance energy,

$$
\begin{aligned}
E^{D_{\alpha}}(G) & =\sum_{i=1}^{n}\left|\partial_{i}-\frac{2 \alpha W(G)}{n}\right|=\sum_{i=1}^{t}\left(\partial_{i}-\frac{2 \alpha W(G)}{n}\right)+\sum_{i=t+1}^{n}\left(\frac{2 \alpha W(G)}{n}-\partial_{i}\right) \\
& =\sum_{i=1}^{t} \partial_{i}-\sum_{i=t+1}^{n} \partial_{i}+\frac{2 \alpha W(G)}{n}(n-2 t)=2 \sum_{i=1}^{t} \partial_{i}-\frac{4 t \alpha W(G)}{n} .
\end{aligned}
$$

We show that

$$
E^{D_{\alpha}}(G)=2 \sum_{i=1}^{t} \partial_{i}-\frac{4 t \alpha W(G)}{n}=\max _{1 \leq i \leq n}\left\{2 M_{i}(G)-\frac{4 i \alpha W(G)}{n}\right\}
$$

where $M_{k}(G)=\sum_{i=1}^{k} \partial_{i}$. Let $s$ be any positive integer such that $1 \leq s \leq n$. For $s \leq t$, we have

$$
M_{t}(G)-M_{s}(G)=\sum_{i=s+1}^{t} \partial_{i} \geq \frac{2 \alpha W(G)}{n}(t-s) .
$$

For $s>t$,

$$
M_{s}(G)-M_{t}(G)=\sum_{i=t+1}^{s} \partial_{i}<\frac{2 \alpha W(G)}{n}(s-t)
$$

that is,

$$
M_{t}(G)-M_{s}(G)>\frac{2 \alpha W(G)}{n}(t-s) .
$$

This shows that, for any value of $s(1 \leq s \leq n)$, we have

$$
M_{t}(G)-M_{s}(G) \geq \frac{2 \alpha W(G)}{n}(t-s)
$$

then

$$
2 M_{t}(G)-\frac{4 t \alpha W(G)}{n} \geq 2 M_{s}(G)-\frac{4 s \alpha W(G)}{n}
$$

which gives the result in Equation (9). Thanks to Lemma 3,

$$
\begin{aligned}
E^{D_{\alpha}}(G) & =\max _{1 \leq i \leq n}\left\{2 M_{i}(G)-\frac{4 i \alpha W(G)}{n}\right\} \\
& \geq 2 M_{1}(G)-\frac{4 \alpha W(G)}{n}=2 \partial_{1}-\frac{4 \alpha W(G)}{n} \\
& \geq \frac{4 W(G)}{n}-\frac{4 \alpha W(G)}{n}=(1-\alpha) \frac{4 W(G)}{n} .
\end{aligned}
$$

Assuming that equality occurs in Equation (8), then equality occurs in Lemma 3 and $t=1$. Since equality occurs in Lemma 3 if and only if $G$ is a transmission regular graph, we see that that equality occurs in Equation (8) if and only if $G$ is a transmission regular graph with $t=1$. Let $G$ be a $k$-transmission regular graph having distance eigenvalues $\delta_{1} \geq \delta_{2} \geq \cdots \geq \delta_{n}$. Then, by Theorem 2 , we have $\partial_{1}=k$ 
and $\partial_{i}=\alpha k+(1-\alpha) \delta_{i}$, for $i=2,3, \ldots, n$. Since $t=1$, it follows that $\partial_{2}<\frac{2 \alpha W(G)}{n}=\alpha k$, which gives $\alpha k+(1-\alpha) \delta_{2}<\alpha k$, in turn giving $\delta_{2}<0$ as $1-\alpha>0$. This implies that the equality holds in Equation (8) if and only if $G$ is a transmission regular graph with a single positive distance eigenvalue.

From Theorem 5, it is clear that for a fixed value of the parameter $\alpha$, among connected graphs $G$ of order $n \geq 2$, the transmission regular graphs with a single positive distance eigenvalue has the minimum generalized distance energy $E^{D_{\alpha}}(G)$.

Since, for a connected graph $G$, we have $\operatorname{Tr}_{i} \geq n-1$, for all $i=1,2, \ldots, n$, it then follows that $2 W(G) \geq n(n-1)$, where the equality holds if and only if $G \cong K_{n}$. The following corollary is an immediate result.

Corollary 1. Let $G$ be a connected graph of order $n \geq 2$ and let $\alpha \in[0,1)$. Then,

$$
E^{D_{\alpha}}(G) \geq 2(1-\alpha)(n-1),
$$

where the equality holds if and only if $G \cong K_{n}$.

Proof. The proof follows from above observation by using Theorem 5 and the fact that $K_{n}$ is a transmission regular graph with a single positive distance eigenvalue.

From Corollary 1 , it is clear that, for a fixed value of the parameter $\alpha$, among all the connected graphs $G$ of order $n \geq 2$, the complete graph $K_{n}$ has the minimum generalized distance energy $E^{D_{\alpha}}(G)$. We note that the conclusion given by Corollary 1 is in agree with the results known for the distance energy obtained by putting $\alpha=0$ [1] and for the distance signless Laplacian energy obtained by putting $\alpha=\frac{1}{2}$ [16].

For a connected graph $G$ of order $n$ and size $m$, we have $\operatorname{Tr}_{i} \geq 2 n-2-d_{i}$, for all $i=1,2, \ldots, n$. It follows that $2 W(G) \geq 2 n(n-1)-2 m$, with equality if and only if $G$ is a graph of diameter at most 2 .

Corollary 2. Let $G$ be a connected graph of order $n \geq 2$ having $m$ edges and let $\alpha \in[0,1)$. Then,

$$
E^{D_{\alpha}}(G) \geq 4(1-\alpha)\left(n-1-\frac{m}{n}\right)
$$

where the equality holds if and only if $G \cong K_{n}$ or $G$ is the cocktail party graph, $G$ is a regular line graph of diameter two, or $G$ is a regular exceptional graph of diameter two.

Proof. Using above observation in Theorem 5 the inequality follows. Equality occurs if and only if $G$ is a transmission regular graph of diameter at most 2 having one positive distance eigenvalue. Since a graph of diameter at most 2 is transmission regular if and only if it is regular, it follows that equality occurs if and only if $G$ is a regular graph of diameter at most 2 having one positive distance eigenvalue. Now, using the discussion before the Corollary 1 of [45], the equality case follows.

Corollary 2 implies that the minimum value of the generalized distance energy $E^{D_{\alpha}}(G)$ is $4(1-$ $\alpha)\left(n-1-\frac{m}{n}\right)$ amongst all connected graphs of order $n$ with $m$ edges. This value is attained by the complete graph $K_{n}$, the cocktail party graph, a regular line graph of diameter 2, and a regular exceptional graph of diameter 2.

The following result gives a lower bound for generalized distance energy $E^{D_{\alpha}}(G)$. The Wiener index $W(G)$ and the transmission degrees are involved. 
Theorem 6. Let $G$ be a connected graph of order $n \geq 3$ and let $\alpha \in[0,1)$. Then,

$$
E^{D_{\alpha}}(G) \geq 2 \sqrt{\frac{\sum_{i=1}^{n}\left(\alpha \operatorname{Tr}_{i}^{2}+(1-\alpha) T_{i}\right)^{2}}{\sum_{i=1}^{n} \operatorname{Tr}_{i}^{2}}}-\frac{4 \alpha W(G)}{n} .
$$

In the case of $\frac{1}{2} \leq \alpha<1$, the equality hold if and only if $G$ is a transmission regular graph with a single positive distance eigenvalue.

Proof. It can be proved similarly by following Lemma 2 and the line of arguments in the proof of the Theorem 5 .

Remark 1. Since all distance regular graphs are transmission regular graphs, by Lemma 9, equality occurs in Theorems 5 and 6 for each of the graphs: a Doob graph, a double odd graph, a Johnson graph, a cocktail party graph, a polygon, a halved cube, a Hamming graph, the Petersen graph, the Schläfli graph, the Gosset graph, the dodecahedron, the icosahedron, and one of the three Chang graphs. It would be inspiring to identify all transmission regular graphs (which are not distance regular) having a single positive distance eigenvalue.

Next, we present a lower bound for the generalized distance energy of a trees via the Wiener index $W(G)$ for $\alpha \in[0,1)$.

Theorem 7. Let $T$ be a tree of order $n \geq 4$ and let $\alpha \in[0,1)$. Then,

$$
E^{D_{\alpha}}(T) \geq(\alpha+2) n-4+\sqrt{(\alpha-2)^{2} n^{2}+4(n-1)(2 \alpha-3)}-\frac{4 \alpha W(T)}{n},
$$

where the equality holds if and only if $T \cong K_{1, n-1}$.

Proof. From the equality in Equation (9), we have

$$
\begin{aligned}
E^{D_{\alpha}}(T) & =\max _{1 \leq i \leq n}\left\{2 M_{i}(T)-\frac{4 i \alpha W(T)}{n}\right\} \\
& \geq 2 M_{1}(T)-\frac{4 \alpha W(T)}{n}=2 \partial_{1}(T)-\frac{4 \alpha W(T)}{n} \\
& \geq(\alpha+2) n-4+\sqrt{(\alpha-2)^{2} n^{2}+4(n-1)(2 \alpha-3)}-\frac{4 \alpha W(T)}{n} .
\end{aligned}
$$

Suppose that the equality holds in Equation (10). Then, equality occurs in Lemma 1 and $t=1$. Equality occurs in Lemma 1 if and only if $G \cong K_{1, n-1}$. For the graph $K_{1, n-1}$, the generalized distance spectrum is $\left\{\alpha(2 n-1)-2^{[n-2]}, \frac{(\alpha+2) n-4 \pm \sqrt{\sigma}}{2}\right\}$, where $\sigma=n^{2} \alpha^{2}-\left(n^{2}+2-2 n\right) 4 \alpha+4\left(n^{2}-3 n+3\right)$ and $2 W\left(K_{1, n-1}\right)=2 n^{2}-4 n+2$. It is now easy to see that $t=1$ for the graph $K_{1, n-1}$. Thus, it follows that equality occurs in Equation (10) if and only if $G \cong K_{1, n-1}$.

For the star graph $K_{1, n-1}$, we have $W\left(K_{1, n-1}\right)=(n-1)^{2}$. Therefore, for any tree $T$ of order $n$, we have $W(T) \geq W\left(K_{1, n-1}\right)=(n-1)^{2}$. The following corollary is an immediate result of Theorem 7 .

Corollary 3. Let $T$ be a tree of order $n \geq 4$ and let $\alpha \in[0,1)$. Then,

$$
E^{D_{\alpha}}(T) \geq(2-3 \alpha) n+8 \alpha-4+\sqrt{(\alpha-2)^{2} n^{2}+4(n-1)(2 \alpha-3)}-\frac{4 \alpha}{n},
$$


where the equality holds if and only if $T \cong K_{1, n-1}$.

By Corollary 3, it is clear that, for fixed $\alpha$, star graph $K_{1, n-1}$ has the minimum generalized distance energy among all the trees of order $n \geq 4$. We note that the conclusion given by Corollary 3 is in agree with the results known for the distance energy obtained by putting $\alpha=0$ [1] and for the distance signless Laplacian energy obtained by putting $\alpha=\frac{1}{2}$ [16].

The following result presents another lower bound for the generalized distance energy $E^{D_{\alpha}}(G)$ again via the Wiener index $W(G)$.

Theorem 8. Let $G$ be connected graph of order $n$ and let $\alpha \in\left[\frac{1}{2}, 1\right]$. Then,

$$
E^{D_{\alpha}}(G) \geq 2(n-1)+2(t-1)(\alpha n-1)-\frac{4 \alpha W(G)}{n},
$$

where the equality holds if and only if $t=1$ and $G \cong K_{n}$.

Proof. Let $G$ be a connected graph of order $n$ having generalized distance eigenvalues $\partial_{1} \geq \partial_{2} \geq \cdots \geq \partial_{n}$. As shown in Theorem 5,

$$
E^{D_{\alpha}}(G)=2 M_{t}(G)-\frac{4 t \alpha W(G)}{n},
$$

where $t$ is the largest positive integer satisfying $\partial_{t} \geq \frac{4 t \alpha W(G)}{n}$ and $M_{t}(G)=\sum_{i=1}^{t} \partial_{i}$. Since $\alpha \in\left[\frac{1}{2}, 1\right]$ and $G$ is a spanning subgraph of $K_{n}$, it follows from Lemma 4 that $\partial(G) \geq \partial\left(K_{n}\right)$, for all $1 \leq i \leq n$. This gives that

$$
M_{t}(G) \geq M_{t}\left(K_{n}\right)=(n-1)+(t-1)(\alpha n-1),
$$

as the generalized distance spectrum of $K_{n}$ is $\left\{n-1, \alpha n-1^{[n-1]}\right\}$. Using Equation (12) in Equation (11), we get

$$
E^{D_{\alpha}}(G) \geq 2(n-1)+2(t-1)(\alpha n-1)-\frac{4 \alpha W(G)}{n} .
$$

Assuming that equality occurs, then there is equality in Equation (12). Since the equality holds in Equation (12) if and only if $G \cong K_{n}$ and $t=1$, it is equivalent to $t=1$ and $G \cong K_{n}$.

The following remark is in order.

Remark 2. As mention in the Introduction, for $\alpha=0$, the generalized distance matrix $D_{\alpha}(G)$ is same as the distance matrix $D(G)$ and, for $\alpha=\frac{1}{2}$, twice the generalized distance matrix $D_{\alpha}(G)$ is same as the distance signless Laplacian matrix $D^{Q}(G)$. Therefore, if in particular we put $\alpha=0$ and $\alpha=\frac{1}{2}$, in all the results obtained in Sections 3 and 4, we obtain the corresponding bounds for the distance energy $E^{D}(G)$ and the distance signless Laplacian energy $E^{Q(G)}$.

\section{Conclusions}

Since the inception of the notion of graph energy [22], it has attracted a significant amount of research attention in chemistry and mathematics. In this paper, we introduce the generalized distance energy $E^{D_{\alpha}}(G)$ of a connected graph $G$. Some new upper and lower bounds are derived based on algebraic graph theory and calculation of eigenvalues. Our results connect some important graph spectrum parameters such as the the Wiener index $W(G)$ as well as the transmission degrees. The extremal graphs in each case 
are delineated. It is found that the minimal generalized distance energy is attained by the complete graphs amongst connected graphs, while it is attained by the star graphs amongst trees.

Author Contributions: Formal analysis, A.A., M.B., H.G. and Y.S.; funding acquisition, Y.S.; Supervision, A.A.; Writing—original draft, A.A., M.B., H.A.G. and Y.S.; Writing—review \& editing, A.A. and Y. S. All authors have read and agreed to the published version of the manuscript.

Funding: Y. Shang was supported in part by the UoA Flexible Fund No. 201920A1001 from Northumbria University.

Acknowledgments: The authors would like to express their deep gratitude to anonymous referees for a careful reading of the paper and a number of helpful suggestions.

Conflicts of Interest: The authors declare no conflict of interest.

\section{References}

1. Aouchiche, M.; Hansen, P. Distance spectra of graphs: A survey. Linear Algebra Appl. 2014, 458, 301-386. [CrossRef]

2. Deza, M.M.; Laurent, M. Geometry of Cuts and Metrics; Volume 15 of Algorithms and Combinatorics; Springer-Verlag: Berlin, Germany, 1997.

3. Engstrom, A. Cut ideals of $k_{4}$-minor free graphs are generated by quadrics. Mich. Math. J. 2011, 60, 705-714. [CrossRef]

4. Martino, I. Vertices collapsing and cuts ideals. Serdica Math. J. 2015, 41, 229-242.

5. Nagel, U.; Petrović, S. Properties of cut ideals associated to ring graphs. J. Commut. Algebra 2009, 1, 547-565. [CrossRef]

6. Alhevaz, A.; Baghipur, M.; Shang, Y. Merging the spectral theories of distance Estrada and distance signless Laplacian Estrada indices of graphs. Mathematics 2019, 7, 995. [CrossRef]

7. Aouchiche, M.; Hansen, P. Two Laplacians for the distance matrix of a graph. Linear Algebra Appl. 2013, 439, 21-33. [CrossRef]

8. Aouchiche, M.; Hansen, P. On the distance signless Laplacian of a graph. Linear Multilinear Algebra 2016, 64, 1113-1123. [CrossRef]

9. Aouchiche, M.; Hansen, P. Some properties of distance Laplacian spectra of a graph. Czechoslov. Math. J. 2014, 64, 751-761. [CrossRef]

10. Alhevaz, A.; Baghipur, M.; Ganie, H.A.; Pirzada, S. Brouwer type conjecture for the eigenvalues of distance signless Laplacian matrix of a graph. Linear Multilinear Algebra 2019. [CrossRef]

11. Alhevaz, A.; Baghipur, M.; Hashemi, E.; Ramane, H.S. On the distance signless Laplacian spectrum of graphs. Bull. Malay. Math. Sci. Soc. 2019, 42, 2603-2621. [CrossRef]

12. Alhevaz, A.; Baghipur, M.; Paul, S. On the distance signless Laplacian spectral radius and the distance signless Laplacian energy of graphs. Discrete Math. Algorithm. Appl. 2018, 10, 1850035. [CrossRef]

13. Aouchiche, M.; Hansen, P. Distance Laplacian eigenvalues and chromatic number in graphs. Filomat 2017, 31, 2545-2555. [CrossRef]

14. Aouchiche, M.; Hansen, P. Cospectrality of graphs with respect to distance matrices. Appl. Math. Comput. 2018, 325, 309-321. [CrossRef]

15. Atik, F.; Panigrahi, P. On the distance spectrum of distance regular graphs. Linear Algebra Appl. 2015, 478, 256-273. [CrossRef]

16. Das, K.C.; Aouchiche, M.; Hansen, P. On (distance) Laplacian energy and (distance) signless Laplacian energy of graphs. Discret. Appl. Math. 2018, 243, 172-185. [CrossRef]

17. Ganie, H.A.; Chat, B.A.; Pirzada, S. On the signless Laplacian energy of a graph and energy of line graph. Linear Algebra Appl. 2018, 544, 306-324. [CrossRef]

18. Pirzada, S.; Ganie, H.A. On the Laplacian eigenvalues of a graph and Laplacian energy. Linear Algebra Appl. 2015, 486, 454-468. [CrossRef]

19. Shang, Y. Distance Estrada index of random graphs. Linear Multilinear Algebra 2015, 63, 466-471. [CrossRef] 
20. Shang, Y. Bounds of distance Estrada index of graphs. Ars Comb. 2016, 128, 287-294.

21. Cui, S.Y.; He, J.X.; Tian, G.X. The generalized distance matrix. Linear Algebra Appl. 2019, 563, 1-23. [CrossRef]

22. Gutman, I. The energy of a graph. Ber. Math. Statist. Sekt. Forschungsz. Graz 1978, 103, 1-22.

23. Gutman, I. The energy of a graph: Old and new results. In Algebraic Combinatorics and Applications; Betten, A., Kohnert, A., Laue, R., Wassermann, A., Eds.; Springer-Verlag: Berlin, Germany, 2001; pp. 196-211.

24. Andrade, E.; Robbiano, M.; Martin, B.S. A lower bound for the energy of symmetric matrices and graphs. Linear Algebra Appl. 2017, 513, 264-275. [CrossRef]

25. Li, X.; Shi, Y.; Gutman, I. Graph Energy; Springer: New York, NY, USA, 2012.

26. Wang, L.; Ma, X. Bounds of graph energy in terms of vertex cover number. Linear Algebra Appl. 2017, 517, $207-216$. [CrossRef]

27. Abreua, N.; Cardoso, D.M.; Gutman, I.; Martins, E.A.; Robbiano, M. Bounds for the signless Laplacian energy. Linear Algebra Appl. 2011, 435, 2365-2374. [CrossRef]

28. Gutman, I.; Zhou, B. Laplacian energy of a graph. Linear Algebra Appl. 2006, 414, 29-37. [CrossRef]

29. Gutman, I.; Furtula, B.; Bozkurt, S.B. On Randić energy. Linear Algebra Appl. 2014, 442, 50-57. [CrossRef]

30. Indulal, G.; Gutman, I.; Vijayakumar, A. On distance energy of graphs. MATCH Commun. Math. Comput. Chem. 2008, 60, 461-472.

31. Nikiforov, V. The energy of graphs and matrices. J. Math. Appl. 2007, 326, 1472-1475. [CrossRef]

32. Nikiforov, V. Beyond graph energy: Norms of graphs and matrices. Linear Algebra Appl. 2016, 506, 82-138. [CrossRef]

33. Shang, Y. Estimating the distance Estrada index. Kuwait J. Sci. 2016, 43, 14-19.

34. Pe na, I.; Rada, J. Energy of digraphs. Linear Multilinear Algebra 2008, 56, 565-579. [CrossRef]

35. Adiga, C.; Balakrishnan, R.; So, W. The skew energy of a digraph. Linear Algebra Appl. 2010, 432, 1825-1835. [CrossRef]

36. Consonni, V.; Todeschini, R. New spectral index for molecule description. MATCH Commun. Math. Comput. Chem. 2008, 60, 3-14.

37. Yang, J.; You, L.; Gutman, I. Bounds on the distance Laplacian energy of graphs. Kragujevac J. Math. 2013, 37, 245-255.

38. Diaz, R.C.; Rojo, O. Sharp upper bounds on the distance energies of a graph. Linear Algebra Appl. 2018, 545, 55-75. [CrossRef]

39. Cui, S.Y.; Tian, G.X.; Zheng, L. On the generalized distance spectral radius of graphs. arXiv 2019, arXiv:1901.07695v1. Preprint.

40. Fan, K. Maximum properties and inequalities for the eigenvalues of completely continuous operators. Proc. Natl. Acad. Sci. USA 1951, 37, 760-766. [CrossRef]

41. Horn, R.; Johnson, C. Matrix Analysis; Cambridge University Press: Cambridge, UK, 1985.

42. Marcus, M.; Minć, H. A Survey of Matrix Theory and Matrix Inequalities; Allyn and Bacon: Boston, MA, USA, 1964.

43. Alhevaz, A.; Baghipur, M.; Pirzada, S. On the bounds for distance signless Laplacian energy-like invariant. 2019, Unpublished work.

44. Koolen, J.H.; Shpectorov, S.V. Distance-regular graphs the distance matrix of which has only one positive eigenvalue. Eur. J. Comb. 1994, 15, 269-275. [CrossRef]

45. Zhou, B.; Ilić, A. On distance spectral radius and distance energy of graphs. MATCH Commun. Math. Comput. Chem. 2010, 64, 261-280.

(C) 2019 by the authors. Licensee MDPI, Basel, Switzerland. This article is an open access article distributed under the terms and conditions of the Creative Commons Attribution (CC BY) license (http:/ / creativecommons.org/licenses/by/4.0/). 